rather unattractive, but relatively cheap, camera-ready production - is a heavy concentration on papers dealing with surfactants, emulsions and microemulsions. Because of the increasing industrial importance of these topics, especially in the oil recovery area, this may be a policy worth pursuing, although in that case a more appropriate title for the journal might be desirable. The quality of papers so far has been maintained at a reasonably high level and several useful short reviews have appeared. Dates of receipt and acceptance of papers are not given so I cannot judge the speed of publication, which is one of the aims of JDST. For those working in the emulsion/surfactant area, a personal subscription to this journal might well be worthwhile.

In the case of CS, well produced and largely typeset conventionally, a much wider range of topics is covered - very like that of JCIS in fact, so that comparisons between the new journal and the established leader in the field are inescapable. So far, it appears that CS is attracting papers which represent a rather more even balance between fundamental and applied aspects of the subject than JCIS, which is mainly concerned with fundamentals. The papers are mostly good, and some excellent contributions have been published. The time from acceptance to publication seems to be mainly in the range seven to ten months. Brief notes (from camera-ready copy) are published more rapidly, together with book reviews and conference announcements.

Whether or not they felt a need for one, colloid scientists will just have to accept the fact that, in Colloids and Surfaces, they now have another broadly-based journal of high quality, which is rapidly becoming indispensable. Persuading a library committee to approve yet another subscription is, of course, a different matter.

John Gregory is Reader in Water Chemistry in the Department of Civil Engineering at University College, University of London. He is Secretary of the Colloid and Surface Chemistry Group, Society of Chemical Industry.

\title{
New Solids: Research for Application
}

\section{Arthur T. Howe}

Solid State Ionics. Principal editor M.S. Whittingham. $9 / \mathrm{yr}$ in 3 vols. (NorthHolland.) DG480. Synthetic Metals. Editor F.L. Vogel. 4/yr. (Elsevier Sequoia.) SwFr.360.

IT WAS known before the turn of the century that the silver ions in solid silver iodide are not static, but behave like a liquid. However this compound, and its derivatives, remained much of a curiosity until the late 1960s when more examples were discovered, heralding the Renaissance of the field which has up till now produced fast ion conductors (sometimes called superionic conductors) for a number of elements in solids - hydrogen, lithium and sodium for example. The potential application of these materials in new batteries has injected an urgency into their study, which prompted the launching of Solid State lonics (SSI) in 1980.

Particularly in the light of long publication times in the Journal of Solid State Chemistry, the more general journal appropriate to the field, SSI has fulfilled its aim of rapid publication, with times for papers typically less than four months in the latest issue, with the special section for notes offering even shorter times. Speed should only be an adjunct of quality, however, and SSI has already attracted an impressive collection of significant papers in the field. In addition the publishers have managed to keep a pleasing, properly printed format. Certainly, I have found a subscription essential, and I am sure that the journal will emerge as the major organ of this important field.

In contrast to the commendable speed of publication in SSI, contributors to Synthetic Metals (SM) currently must wait a considerable time - 10 to 18 months to see their papers in print. Nonetheless this journal, too, looks set for a healthy future. SM is well produced and, as well as publishing papers and reviews, frequently features collections of papers presented at conferences.

The journal was designed to highlight the growth of interest in the areas of intercalation compounds of graphite, transition metal compounds and quasi onedimensional conductors. The term "synthetic metals" was coined to describe those materials which possessed metallic conductivity but were not the normal elemental metals. Conducting films of polyacetylene, $(\mathrm{CH})_{\ngtr}$, first synthesized in 1974 , sparked off the search for plastic conductors to replace copper and aluminium. SM has provided a muchneeded forum for the explosion of activity in this truly multidisciplinary area - for example, the spread of the journal is indicated by reports in a recent issue on Raman, ion implantation, microscopic, thermopower and conductivity experiments.

Arthur T. Howe is at the Amoco Research Center, Naperville, Illinois.

\section{Angles on Surfaces}

\section{D.P. Woodruff}

Surface Science Letters. Editor H.C. Gatos. 24/yr. (North-Holland.) $\$ 30$ (personal only). Surface and Interface Analysis. Editor-in-chief D. Briggs. 6/yr. (Heyden.) £82, \$180, DM 382.

WHIIE much of the literature on fundamental studies of surfaces is dispersed through a variety of journals, Surface Science has established itself as the mainstream journal for such work and has expanded its scale to a volume (in two parts) of about 700 pages each month. While this journal has long had a "letters" section, their publication has not been especially speedy and the cost of the journal lies far outside the budget of individual researchers. Surface Science Letters is not a new journal as such, but an attempt to revitalize the letters section of its parent by making this section available to private subscribers at relatively modest cost. As such it is a very worthwhile venture; publication delays are still a little long ( 6 months or so) but the availability of this new format is encouraging the convergence of more short papers and speculative ideas into a single periodical which is guaranteed a large audience.

By contrast, Surface and Interface Analysis (SIA) is an entirely new journal of modest proportions (each issue is about 40 large-format pages) which attempts to fill a new position in the literature. Many of the new techniques which have emerged in the past 15 years of fundamental surface science have proved to be of considerable value in the elucidation and characterization of materials' problems related to surface and thin films (including interface) phenomena. The publication of work on these applications has been dispersed in a range of journals. To some extent, this situation has advantages; the specific application may be of far more interest to those concerned with the relevant material (for example, glasses, catalysts, semiconductor devices) than to those applying the same technique to different systems. On the other hand, many problems are common to the technique quantification, instrumentation and calibration, for example - and a common forum for these is clearly valuable. SIA is attracting such papers along with a number of contributions on specific applications.

Undoubtedly the journal does fill a need, although the size of this valuable core of literature is small and could not support a larger journal. In principle, another relatively recent journal, Applications of Surface Science, has a similar objective; in practice, however, it has failed to distinguish itself sufficiently in content from its sister journal, Surface Science.

D.P. Woodruff is in the Physics Department at the University of Warwick. 